\title{
Retrospective exposure assessment for a cohort study into respiratory effects of acid anhydrides
}

\author{
M J A van Tongeren, R D Barker, K Gardiner, J M Harris, K M Venables, J M Harrington, \\ A J Newman Taylor
}

\begin{abstract}
Objectives-To estimate past exposure to phthalic (PA), trimellitic (TMA) and maleic anhydride (MA) in three alkyd resin and one cushioned flooring factory to estimate exposure-response relations in a retrospective cohort study.

Methods-Personal exposure measurements were carried out in 1992 and quantitative and qualitative information on past exposure and production processes were collected. Job titles were ranked by decreasing exposure and amalgamated into job categories and exposure groups. Multiplication factors for back calculating past exposure levels were estimated with past exposure data, or if no such data were available these factors were estimated by a panel of occupational hygienists. Exposure levels were back calculated starting with the exposure levels in 1992.
\end{abstract}

Results-High exposures to PA were estimated to have occurred among workers operating the PA melting pots in factory 1 (estimated exposure in 1960-9 was 2480 $\left.\mu \mathrm{g} . \mathrm{m}^{-3}\right)$. Highest concentrations of TMA were estimated to have occurred among the ink mixers in factory 2 from 1979 to $1986\left(554 \mu \mathrm{g} \cdot \mathrm{m}^{-3}\right)$. Exposure in most other job titles was thought to be fairly constant over time for PA, TMA, and MA.

Conclusions-Exposure to acid anhydride at these factories has fallen during the period covered by the study. However, it is estimated that in only one job in factory 2 did past exposure to acid anhydride exceed the current occupational exposure standard. Accuracy of the estimated exposure is limited by a paucity of reliable past exposure data.

(Occup Environ Med 1998;55:692-696)

Keywords: acid anhydrides; retrospective exposure assessment; occupational asthma

Acid anhydrides are respiratory sensitisers. ${ }^{1}$ Previous publications have suggested that occupational asthma may be caused by phthalic anhydride (PA), ${ }^{2-4}$ trimellitic anhydride (TMA), ${ }^{5}$ maleic anhydride (MA), ${ }^{7}$ and several other acid anhydrides. ${ }^{1}$ However, a quantitative relation between exposure and sensitisation or occupational asthma has not been established as exposure data have either not been available or have lacked sufficient detail.

To investigate whether a relation exists between exposure to acid anhydrides and sen- sitisation, respiratory symptoms, and bronchial hyperresponsiveness, a retrospective cohort study was carried out. The cohort consisted of both current and past employees of four factories where acid anhydrides had been used. Three factories principally produced alkyd resins (factory 1, 3, and 4) whereas the fourth (factory 2) produced cushioned flooring. Exposure to acid anhydride was assessed in each of the factories extending back to the inception dates for the cohorts, which varied between the factories. ${ }^{8}$ Factories 1,3 , and 4 use or had used PA, TMA, and MA, whereas factory 2 only ever used TMA.

The study design, health outcomes, and results of the personal exposure measurements made in 1992 are reported elsewhere. ${ }^{89}$ The study design required both an assessment of full shift exposure to acid anhydride for each worker at each time during the period covered by the cohort and also an estimate of cumulative exposure for each worker. To achieve this, exposure matrices were developed with job and time as the axes (job-time-exposure matrices). The methods and results of the retrospective exposure assessment that allowed these measures to be obtained are presented in this paper.

\section{Methods and materials}

The job-time-exposure matrices were developed in four steps. Firstly, the current exposure in 1992 was measured. ${ }^{9}$ Secondly, exposure was estimated for job titles with insufficient current exposure measurements. Next, multiplication factors were established for the retrospective exposure assessment with past exposure data and qualitative information. Finally, exposure estimates in the job-time-exposure matrices were calculated.

\section{CURRENT EXPOSURE MEASUREMENTS}

Personal full shift exposure measurements for PA, TMA, and MA were carried out in the four factories in 1992. The measurements were made during planned factory visits that lasted 2-4 weeks. Full shift samples were obtained from about one in every 10 shifts worked during visits to the factories. Within each job title, workers were selected randomly to wear a personal monitor during a 2-4 week sampling period. Details of the methods and materials of these measurements are reported elsewhere. ${ }^{9}$

BASELINE EXPOSURE ESTIMATES

Ninety five job titles were identified within the cohort. As baseline for the job time exposure matrices, estimates of current average full shift exposure for each of these job titles were 
Table 1 Estimated baseline exposure to phthalic anhydride (PA) by job title, job category, and exposure group

\begin{tabular}{|c|c|c|c|c|}
\hline $\begin{array}{c}\text { Exposure group } \\
\text { Fob category } \\
\text { fob title }\end{array}$ & Factory & $\begin{array}{l}\text { Samples } \\
\text { (n) }\end{array}$ & $\begin{array}{l}\text { Mean } \\
\left(\mu g . m^{-3}\right)\end{array}$ & $\begin{array}{l}\text { Baseline } \\
\text { exposure } \\
\left(\mu g . m^{-3}\right)\end{array}$ \\
\hline High exposure & & 12 & 138 & \\
\hline Resin operators solid PA & & 12 & 138 & \\
\hline Resin operator B & 1 & - & - & 138 \\
\hline Resin operator A & 3 & 12 & 138 & 138 \\
\hline Resin operator B & 3 & - & - & 138 \\
\hline Medium exposed & & 30 & 20.1 & \\
\hline Resin operators liquid PA & & 22 & 22.2 & \\
\hline Resin operator A & 1 & 9 & 25.1 & 25.1 \\
\hline Resin operator C & 1 & - & - & 22.2 \\
\hline Resin operator A & 4 & 13 & 20.1 & 20.1 \\
\hline Resin operator B & 4 & - & - & 22.2 \\
\hline Filter/lab operators factory 3 & & 5 & 17.3 & \\
\hline Filter operator & 3 & 3 & 19.4 & 17.3 \\
\hline Research and development lab assistant & 3 & 2 & 16.6 & 17.3 \\
\hline Pilot plant operators & & 3 & 10.4 & - \\
\hline Pilot plant operator & 4 & 3 & 10.4 & 20.1 \\
\hline Pilot plant operator & 3 & - & - & 20.1 \\
\hline Low exposure & & 41 & 2.2 & \\
\hline Not exposure & & 22 & 1.3 & \\
\hline
\end{tabular}

ॠ Estimated exposure to be used for starting point of back calculation of exposure.

Table 2 Estimated baseline exposure to trimellitic anhydride (TMA) by job title, job category and exposure group

\begin{tabular}{lllll}
\hline $\begin{array}{l}\text { Exposure group } \\
\text { fob category } \\
\text { fob title }\end{array}$ & Factory & $\begin{array}{l}\text { Samples } \\
(n)\end{array}$ & $\begin{array}{l}\text { Mean } \\
\left(\mu g . m^{-3}\right)\end{array}$ & $\begin{array}{l}\text { Baseline } \\
\text { exposure } \\
\left(\mu g . m^{-3}\right)\end{array}$ \\
\hline High exposure & & 8 & 15.4 & \\
$\quad$ Ink mixer or colour matcher & 2 & 8 & 15.4 & \\
$\quad$ Ink mixer & 2 & 4 & 18.3 & 15.4 \\
$\quad$ Colour matcher & 4 & 12.5 & 15.4 \\
$\quad$ Medium expourse & 24 & 4.9 & \\
Printing & 2 & 24 & 4.9 & \\
$\quad$ Printing operator & 2 & 6 & 5.4 & 5.4 \\
$\quad$ Reel operator & 2 & 8 & 5.7 & 5.7 \\
$\quad$ Pilot plant operator or supervisor & & 73 & 0.8 & 3.6 \\
Low exposure & & 23 & 0.5 & \\
Not exposure & & &
\end{tabular}

^Estimated exposure to be used for starting point of back calculation of exposure.

required. When five or more current exposure measurements were available for a job title, the arithmetic mean of these measurements was used directly in the matrix. In some cases there were insufficient measurements to make an accurate assessment of the average exposure in a particular job title, especially as some job titles did not exist at the time of sampling. Therefore, all job titles were amalgamated into job categories based on similarities in work activities and production processes. The arithmetic mean for all full shift exposure measurements within a job category was assigned to a job title if less then five measurements were available for that job title.
An example of where no exposure measurements were available was for a resin operator B in factory 1 , as the job title did not exist during the time of the exposure measurements. However, the job description was similar to that of resin operator A in factory 3. In both job titles a considerable part of the activities was taken up by loading solid PA into the reactors. Therefore, the resin operator B in factory 1 was given the average exposure of job category "resin operators solid PA" as the baseline exposure estimate (table 1).

Even after grouping job titles into job categories there were still occasionally less than five exposure measurements available. For this reason job categories were merged into exposure groups on the basis of the exposure measurements and in some cases on work activities and production processes. If less than five measurements were available for a job category, the average of the exposure group was used as an estimate of the baseline exposure estimate of the job title. For example, only three PA exposure measurements were available for the job category pilot plant operators and therefore the job titles in this category were assigned the average of the medium exposure group (table 1).

\section{MULTIPLICATION FACTORS}

Qualitative information was gathered on past working practices and production processes with records on working procedures, job descriptions, purchase and production, maintenance, accidents and incidents, and records held by the Occupational Health and Safety departments. Also, several long term employees, managers, and occupational health, hygiene, and safety personnel were interviewed with a standard questionnaire. A document was written for each factory containing all of the relevant information, which was subsequently reviewed for completeness and accuracy by factory staff.

All past exposure data available from the factories were collected and entered in a data base. Past exposure data were sparse, collected with varying sampling and analytical techniques, and generally not collected as part of a systematic sampling regime, but taken mainly during worst case situations for compliance purposes. Direct exposure estimates from past

Table 3 fob-time-exposure matrix for phthalic anhydride (PA) in factory 1

\begin{tabular}{|c|c|c|c|c|c|c|c|c|c|}
\hline Fob title & $1960-9$ & $1970-7$ & $1978-81$ & $1982-5$ & 1986 & 1987 & $1988-9$ & $1990-1$ & 1992 \\
\hline Resin operator A & & & & 25.1 & 25.1 & 52.7 & 50.2 & 50.2 & 25.1 \\
\hline Resin operator B & 2480 & 413 & 207 & 207 & 138 & 138 & 34.4 & & \\
\hline Resin operator C & & & & 22.2 & 22.2 & 22.2 & 22.2 & & \\
\hline Resin tester & 1.8 & 1.8 & 1.8 & 1.8 & 1.8 & 1.8 & 1.8 & & \\
\hline Filter operator A & & & & 1.5 & 1.5 & 1.5 & 1.5 & 1.5 & 1.5 \\
\hline Filter operator B & 1.5 & 1.5 & 1.5 & 1.5 & 1.5 & 1.5 & 0.4 & & \\
\hline Warehouseman A & & & & 2.2 & 2.2 & 4.4 & 2.2 & 2.2 & 2.2 \\
\hline Warehouseman B & 2.2 & 2.2 & 2.2 & 2.2 & 2.2 & 2.2 & 2.2 & & \\
\hline $\begin{array}{l}\text { Research and development lab } \\
\text { assistant }\end{array}$ & & & & 2.2 & 2.2 & 2.2 & 2.2 & 2.2 & 2.2 \\
\hline Fitter A & & & & 4.4 & 4.4 & 8.8 & 2.2 & 2.2 & 2.2 \\
\hline Electrician A & & & & 2.2 & 2.2 & 2.2 & 2.2 & 2.2 & 2.2 \\
\hline Fitter B & 2.2 & 2.2 & 2.2 & 2.2 & 2.2 & 2.2 & 2.2 & & \\
\hline Electrician B & 2.2 & 2.2 & 2.2 & 2.2 & 2.2 & 2.2 & 2.2 & & \\
\hline Supervisor A & & & & 2.2 & 2.2 & 2.2 & 2.2 & 2.2 & 2.2 \\
\hline Supervisor B & 2.2 & 2.2 & 2.2 & 2.2 & 2.2 & 2.2 & 2.2 & & \\
\hline
\end{tabular}

Exposure is given in $\mu \mathrm{g} \cdot \mathrm{m}^{-3}$. 
Table 4 Fob-time-exposure matrix for phthalic anhydride (PA) in factory 3

\begin{tabular}{llll}
\hline fob title & $1979-89$ & 1990 & $1991-2$ \\
\hline Resin operator A & 138 & 138 & 138 \\
Resin operator B & 138 & 138 & 138 \\
Pilot plant operator & - & 20.1 & 20.1 \\
Filter operator & 17.3 & 17.3 & 17.3 \\
Warehouseman & 2.2 & 2.2 & 2.2 \\
Resin tester & 2.2 & 2.2 & 2.2 \\
Research and development & & & \\
$\quad$ lab assistant & 26.0 & 26.0 & 17.3 \\
Fitter & 2.2 & 2.2 & 2.2 \\
Electrician & 2.2 & 2.2 & 2.2 \\
Supervisor & 2.2 & 2.2 & 2.2 \\
\end{tabular}

Exposure is given in $\mu \mathrm{g} \cdot \mathrm{m}^{-3}$.

exposure data were not valid as these data are not representative of the total exposure distribution.

A panel of three occupational hygienists with experience in estimating past exposure, reached a consensus on the events in the past which were likely to have affected exposure to acid anhydrides. After this selection, multiplication factors were estimated by taking the ratio of past exposure data collected by the factories before and after the exposure modifying event.

Occasionally, multiplication factors could not be derived directly from past exposure data. In these cases, exposure was either assumed to be similar to other job titles with a known exposure or the multiplication factors were estimated by the occupational hygiene panel. For example, in 1987 resin operators A in factory 1 loaded solid PA in the reactor vessels during 3 months of the year instead of operating an automatic liquid PA system. It was assumed that the exposure during that period was similar to that measured in 1992 among resin operators loading solid PA in factory 3. The multiplication factor was estimated subse- quently by taking the ratio of the exposure of the resin operators who loaded solid PA with the exposure of resin operators who worked with liquid PA (138/25 5 5.5). However, during the remaining three quarters of the year liquid PA was used and therefore, the multiplication factor was adjusted accordingly $(5.5+3) / 4 \approx 2$.

If comparison with other job titles with exposure data was not possible, the panel estimated the multiplication factor based on the qualitative information. For example, in factory 4, a laminar flow booth for weighing acid anhydrides was installed in 1984 and local exhaust ventilation on the manholes of the reactors was installed in the pilot plant in 1991. Both control measures were estimated to reduce exposure by a factor of 10 . However, because weighing and loading of PA only took place for respectively, $5 \%$ and $10 \%$ of the working time the multiplication factors were modified to $1.5(10 \times 0.05+0.95=1.45)$ and 2 $(10 \times 0.10+0.90=1.90)$, respectively.

Finally, past exposure was estimated starting with current exposure and calculating backwards with the multiplication factors. In general, the baseline exposure was either the exposure in 1992, when the exposure measurements were carried out, or the exposure just before the job title ended. In some cases, however, the baseline was the exposure some time before the job title ended, due to a cut down of activities. For example, in 1988, two years before closure, production in the factory where resin operators B of factory 1 worked, production was reduced by $50 \%$ and the use of PA almost stopped. In this situation, the baseline exposure was the estimated exposure before production was cut in 1988 and the exposure was calculated both backwards and forwards in time. The results of the retrospective exposure

Table 5 fob-time-exposure matrix for phthalic anhydride (PA) in factory 4

\begin{tabular}{lrrrrrrr}
\hline fob title & $1960-7$ & 1968 & $1969-77$ & 1978 & $1979-83$ & $1984-90$ & $1991-2$ \\
\hline Resin operator A & & 30.2 & 30.2 & 20.1 & 20.1 & 20.1 & 20.1 \\
Resin operator B & 44.4 & 44.4 & 44.4 & 44.4 & 22.2 & & 2.2 \\
Warehouseman & 4.9 & 4.9 & 7.3 & 7.3 & 4.4 & 2.2 & 20.1 \\
Pilot plant operator & 60.3 & 60.3 & 60.3 & 60.3 & 60.3 & 40.2 & 1.8 \\
Resin tester & 1.8 & 1.8 & 1.8 & 1.8 & 1.8 & 1.8 & 1.5 \\
Filter operator & 1.5 & 1.5 & 1.5 & 1.5 & 1.5 & 2.2 & 2.2 \\
Fitter & 4.9 & 4.9 & 7.3 & 7.3 & 4.4 & 2.2 & 2.2 \\
Electrician & 4.9 & 4.9 & 7.3 & 7.3 & 4.4 & 2.2 & 2.2 \\
Supervisor A & 2.2 & 2.2 & 2.2 & 2.2 & 2.2 & 2.2 & 2.2 \\
Supervisor B & 4.9 & 4.9 & 7.3 & 7.3 & 4.4 & & 2.2 \\
\hline
\end{tabular}

Exposure is given in $\mu \mathrm{g} \cdot \mathrm{m}^{-3}$.

Table 6 fob-time-exposure matrix for trimellitic anhydride (TMA) in factory 1

\begin{tabular}{|c|c|c|c|c|c|c|}
\hline fob title & $1960-71$ & $1972-5$ & $1976-81$ & $1982-7$ & $1988-9$ & $1990-2$ \\
\hline Resin operator A & & & & 0.8 & 0.8 & 0.8 \\
\hline Resin operator B & 0 & 0.2 & 0.7 & 0.7 & 0.2 & \\
\hline Resin operator C & & & & 0.7 & 0.7 & \\
\hline Resin tester & 0 & 0.8 & 0.8 & 0.8 & 0.8 & \\
\hline Filter operator A & & & & 0.6 & 0.6 & 0.6 \\
\hline Filter operator B & 0 & 0.6 & 0.6 & 0.6 & 0.2 & \\
\hline Warehouseman A & & & & 0.7 & 0.7 & 0.7 \\
\hline Warehouseman B & 0 & 0.1 & 0.7 & 0.7 & 0.2 & \\
\hline Research and development lab assistant & & & & 0.5 & 0.5 & 0.5 \\
\hline Fitter A & & & & 1.8 & 1.8 & 1.8 \\
\hline Electrician A & & & & 0.8 & 0.8 & 0.8 \\
\hline Fitter B & 0 & 1.4 & 1.4 & 1.4 & 1.4 & \\
\hline Electrician B & 0 & 0.8 & 0.8 & 0.8 & 0.8 & \\
\hline Supervisor A & & & & 0.8 & 0.8 & 0.8 \\
\hline Supervisor B & 0 & 0.8 & 0.8 & 0.8 & 0.8 & \\
\hline
\end{tabular}

Exposure is given in $\mu \mathrm{g} \cdot \mathrm{m}^{-3}$. 
Table 7 fob-time-exposure matrix for trimellitic anhydride (TMA) in factory 2

\begin{tabular}{lcccc}
\hline Fob title & $1979-86$ & $1987-8$ & $1989-90$ & $1991-2$ \\
\hline Printing operator & 10.8 & 10.8 & 5.4 & 5.4 \\
Reel operator & 11.4 & 11.4 & 5.7 & 5.7 \\
Ink mixer & 554 & 92.4 & 15.4 & 15.4 \\
Colour matcher & 15.4 & 15.4 & 15.4 & 15.4 \\
Plate or proof operator & 3.6 & 3.6 & 3.6 & 3.6 \\
Plate or proof supervisor & 5.4 & 5.4 & 5.4 & 3.6 \\
Electrician & 0.8 & 0.8 & 0.8 & 0.8 \\
Fitter & 0.8 & 0.8 & 0.8 & 0.8 \\
\hline
\end{tabular}

Exposure is given in $\mu \mathrm{g} \cdot \mathrm{m}^{-3}$.

Table 8 fob-time-exposure matrix for trimellitic anhydride $(T M A)$ in factory 3

\begin{tabular}{lllll}
\hline fob title & $1979-86$ & $1987-9$ & 1990 & $1991-2$ \\
\hline Resin operator A & 0 & 0 & 0 & 0 \\
Resin operator B & 0 & 0.7 & 0.7 & 0.7 \\
Pilot plant operator & $\overline{0}$ & $\overline{0.6}$ & 0.8 & 0.8 \\
Filter operator & 0 & 0.6 & 0.6 & 0.6 \\
Warehouseman & 0 & 1.4 & 0.7 & 0.7 \\
Resin tester & 0.8 & 0.8 & 0.8 & 0.8 \\
Research and & & & & \\
$\quad$ development lab & & & & \\
$\quad$ assistant & 0.6 & 0.6 & 0.6 & 0.5 \\
Fitter & 0.9 & 1.4 & 1.4 & 1.4 \\
Electrician & 0.8 & 0.8 & 0.8 & 0.8 \\
Supervisor & 0.8 & 0.8 & 0.8 & 0.8 \\
\end{tabular}

Exposure is given in $\mu \mathrm{g} \cdot \mathrm{m}^{-3}$.

assessment were sent for review and comments to the occupational health, hygiene, and safety personnel of the factories.

\section{Results}

Results of the exposure measurements made in 1992 are reported in detail elsewhere ${ }^{9}$ and are summarised for several job titles in tables 1 and 2 for PA and TMA, respectively. The last column in these tables gives the exposure estimates that were used as starting points for back calculations in the retrospective exposure assessment (baseline exposure).

For PA the high exposure group consisted of resin operators who loaded solid PA into reactor kettles (mean $138 \mu \mathrm{g} . \mathrm{m}^{-3}$ ), whereas resin operators that worked with liquid PA are in the medium exposure group (table 1). Pilot plant operators in the resin manufacturing factories, filter operators, and research and development laboratory assistants in factory 3 also formed part of the medium exposure group (mean $20.1 \mu \mathrm{g} \cdot \mathrm{m}^{-3}$ ). The low exposure group (mean $2.2 \mu \mathrm{g} \cdot \mathrm{m}^{-3}$ ) was formed by warehousemen, fitters, filter operators, laboratory personnel, supervisors, and electricians. No PA was used in factory 2 and therefore all job titles were assigned to the no exposure group.

High and medium exposures to TMA were found only in the cushioned flooring factory (table 2). The TMA was used daily at this fac-

Table 9 fob-time-exposure matrix for trimellitic anhyddride (TMA) in factory 4

\begin{tabular}{llllll}
\hline fob title & $1960-72$ & $1973-7$ & $1978-83$ & $1984-90$ & $1991-2$ \\
\hline Resin operator A & - & 0 & 0 & 0 & 0 \\
Resin operator B & 0 & 7.7 & 0.7 & - & - \\
Warehouseman & 0 & 0.7 & 0.7 & 0 & 0 \\
Pilot plant operator & 0.9 & 0.9 & 0.9 & 0.9 & 0.8 \\
Resin tester & 0 & 0 & 0 & 0 & 0 \\
Filter operator & 0 & 0.6 & 0.6 & 0.6 & 0.6 \\
Fitter & 0 & 1.4 & 1.4 & 0 & 0 \\
Electrician & 0 & 0.8 & 0.8 & 0 & 0 \\
Supervisor A & 0 & 0.8 & 0.8 & 0 & 0 \\
Supervisor B & 0 & 0 & 0 & 0 & 0 \\
\hline
\end{tabular}

Exposure is given in $\mu \mathrm{g} \cdot \mathrm{m}^{-3}$. tory, but only occasionally in the other factories. The high exposure group was formed by the ink and colour mixers (mean $15.4 \mu \mathrm{g} \cdot \mathrm{m}^{-3}$ ), and the workers involved with the printing of the floor covering formed the medium exposure group (mean $4.9 \mu \mathrm{g} \cdot \mathrm{m}^{-3}$ ). All other job titles were assigned to the low or no exposure groups. At factory 2 some exposure measurements were made when the production line had broken down. During this period the printers experienced unusually high exposures to TMA while chipping inks off the factory floor. As this was the only time in the history of the factory that this activity had taken place the associated exposure measurements were not used in the job-time-exposure matrix.

For exposure to MA all job titles were assigned to the low (mean $2.2 \mu \mathrm{g} \cdot \mathrm{m}^{-3}$ ) or no exposure groups. Again, all job titles in factory 2 were considered not to be exposed to MA. Although there are some differences in the exposure between the job categories, MA was seldom used in all factories and therefore average exposure was thought to be low for all categories.

Tables 3-9 give the job-time-exposure matrices by factory for exposure to PA and TMA, respectively. No matrices are shown for exposure to MA as exposure was estimated to be fairly constant across job titles and time. No matrix for PA exposure in factory 2 is given as this anhydride was never used in this factory. Highest estimated exposure to PA occurred between 1960 and 1969 among resin operators $\mathrm{B}$ in factory $1\left(2480 \mu \mathrm{g} \cdot \mathrm{m}^{-3}\right)$. These workers melted PA in open melting pots. No major changes in the production process or ventilation systems occurred in factory 3, and apart from the research and development laboratory assistants, average exposure levels were estimated to have remained unchanged since 1979. The PA exposure in the 1960s was higher among resin and pilot plant operators in factory 1 than among those in factory 4 . This was because liquid PA had always been used in an enclosed system in factory 4 .

Highest exposure to TMA was estimated to have occurred among the ink mixers (factory 2) from 1979 to $1986\left(554 \mu \mathrm{g} \cdot \mathrm{m}^{-3}\right)$. No suitable respiratory protective equipment was used during these years. Also, training of the ink mixers in handling TMA and improvement of the local exhaust ventilation system on the TMA mixer were introduced in 1987 and 1989, respectively. Improved air extraction in the printing area reduced exposure levels for printing and reel operators. In all other factories exposure to TMA was low.

\section{Discussion}

This paper describes the approach and results of a retrospective exposure assessment for PA, TMA, and MA in a study of the respiratory effects of these anhydrides. A retrospective exposure assessment was necessary as we were particularly interested in exposure to acid anhydrides at the time of sensitisation and at the onset of respiratory symptoms. ${ }^{8}$ The retrospective cohort study design was chosen because in a cross sectional study any possible 
exposure-response relation is likely to remain undetected due to survivor bias. The main aim was to assess the relation between exposure to acid anhydrides and consequent respiratory effects.

Numerous methods for estimating past exposure have been reported..$^{10-13}$ Unfortunately, most require a substantial amount of reliable exposure data or depend on measurements during simulation of past processes. If no or little past exposure data are available, qualitative information can be used to assist in a retrospective exposure assessment. ${ }^{14}$

The method for retrospective exposure assessment described in this paper relied on estimating current exposure levels and multiplication factors to back calculate the past exposure. These factors were obtained from past exposure data or were estimated by the occupational hygiene panel. The results from the retrospective exposure assessment suggest that the estimated exposure levels in the past were sometimes much higher than those measured in 1992. However, exposure among job titles not directly involved in the production process was not thought to have changed much.

The method for retrospective exposure assessment described in this paper has several disadvantages. If less than five exposure measurements were available for a job title, the arithmetic mean exposure of the relevant job category or exposure group was used as the baseline exposure from which past exposures were calculated. It is well known that exposure can vary enormously between workers and over time even within the same jobs, ${ }^{15}$ and therefore misclassification could have occurred. Furthermore, the multiplication factors often had to be estimated by a panel of occupational hygienists, who only had qualitative information to rely on. Available past exposure data, which were collected during worst case scenarios or particular tasks, were used for the calculation of multiplication factors. It was assumed that, although the past exposure data were not representative of the total exposure distribution, the ratio of exposure before and after a modifying event was representative for the change in the average exposure. Due to a lack of reliable full shift past exposure data, it was not possible to confirm or validate the estimated exposures.

Although every effort was made to ensure that the histories of the factories were as complete as possible, it is unlikely that all factors that modified exposure were taken into account. Collection of information on past exposure relied heavily on factory records, which as a rule are not intended for this purpose, and anecdotal information from long term employees. An amalgam of the information collected from the factories was fed back to ensure that it was as complete and accurate as possible. However, factors such as improvement in housekeeping and manual handling could not be taken into account, as these were introduced gradually and no records of these measures had been kept. It is therefore possible that our estimates of past exposure are inaccurate, particularly for workers at factories 1 and 4 in the 1960s. At factories 2 and 3 the cohort inception was 1979 and there had been relatively few changes in the production process in that time.

The retrospective exposure assessment is based on an extensive series of measurements of exposure to acid anhydride. Furthermore, estimation of past exposure was carried out through the development of multiplication factors, rather than direct subjective assessment. Whenever possible these multiplication factors were based on actual exposure data from the companies. In other cases, these factors were estimated by an experienced occupational hygiene panel based on information on the working practices and production processes. All this information was fed back to the companies to ensure accuracy and completeness. Finally, the assessments were carried out without knowledge of the health status of the study subjects.

Therefore, we are confident that the use of job-time-exposure matrices will not cause artefactual exposure-response relations. Also, we think that any non-differential misclassification has been kept to a minimum given the data. We think that considerably more misclassification would have occurred, both in actual level of exposure and in the ranking of the job titles, if no retrospective exposure assessment had been carried out.

The study was funded by the Health and Safety Executive and the Royal Society. We thank the participating factories and their employees.

1 Venables KM. Low molecular weight chemicals, hypersensitivity, and direct toxicity: the acid anhydrides. Br f Ind Med 1989;46:222-32.

2 Kern RA. Asthma and allergic rhinitis due to sensitisation to phthalic acid. I Allergy 1939;10:164-5.

3 Maccia CA, Bernstein L, Emmet EA, et al. In vitro demonstration of specific IgE in phthalic anhydride hypersensitivity. Am Rev Respir Dis 1976;113:701-4.

4 Wernfors $M$, Nielson J, Schutz A, et al. Phthalic anhydride induced occupational asthma. Int Arch Allergy Appl Immunol 1986;79:77-82.

5 Zeiss CR, Patterson R, Pruzansky JJ, et al. Trimellitic nhydride-induced airway syndromes: clinical and immunologic studies. F Allergy Clin Immunol 1977;60:96-103.

6 Sale SR, Roach D, Zeiss CR, et al. Clinical and immunologic correlations in trimellitic anhydride airway syndromes. $\mathcal{F}$ Allergy Clin Immunol 1981;68:183-93.

7 Lee HS, Wang YT, Cheong TH, et al. Occupational asthma due to maleic anhydride. Br F Ind Med 1991;48:283-5.

8 Barker RD, van Tongeren MJA, Harris JM, et al. Risk factors for sensitisation and respiratory symptoms among workers for sensitisation and respiratory symptoms among workers 1998;55:684-91.

9 van Tongeren MJA, Barker RD, Gardiner K, et al. Exposure to acid anhydrides in three resin and one cushioned flooring manufacturing plants. Ann Occup Hyg 1995;39:559-71.

10 Cherrie J, Krantz S, Schneider T, et al. An experimental simulation of an early rock wool/slag wool production process. Ann Occup Hyg 1987;31:583-93.

11 Dosemeci M, Stewart PA, Blair A. Three proposals for retrospective, semiquantitative exposure assessments and their comparison with the other assessment methods. Appl Occup Environ Hyg 1990;5:52-9.

12 Hornung RW, Greife AL, Stayner LT, et al. Statistical model for prediction of retrospective exposure to ethylene oxide in an occupational mortality study. Am f Ind Med 1994;25: 825-36.

13 Yu RC, Tan W-Y, Mathew RM, et al. A deterministic mathematical model for quantitative estimation of historical exposure. Am Ind Hyg Assoc f 1990;51:194-201.

14 Stewart PA, Blair A, Dosemeci M, et al. Collection of exposure data for retrospective occupational epidemiologic studies. Appl Occup Environ Hyg 1991;6:280-9.

15 Kromhout H, Symanski E, Rappaport SM. A comprehensive evaluation of within and between worker components of occupational exposure to chemical agents. Ann Occup Hyg 1993;37:253-70. 